\title{
Fast Background Elimination in Fluorescence Microbiology Images: Comparison of Four Algorithms *
}

\author{
Shan Gong and Antonio Artés-Rodríguez \\ Department of Signal Theory and Communications, \\ Universidad de Carlos III de Madrid, \\ \{concha, antonio\}@tsc.uc3m.es
}

\begin{abstract}
In this work, we investigate a fast background elimination front-end of an automatic bacilli detection system. This background eliminating system consists of a feature descriptor followed by a linear-SVMs classifier. Four state-of-the-art feature extraction algorithms are analyzed and modified. Extensive experiments have been made on real sputum fluorescence images and the results reveal that $96.92 \%$ of the background content can be correctly removed from one image with an acceptable computational complexity.
\end{abstract}

Keywords: Tuberculosis bacilli, background remove, object detection, fluorescence image.

\section{Introduction}

Tuberculosis is a contagious disease that causes thousands of death every year around the whole word. Sputum specimens are firstly stained with fluorescent dye and fluorescence images can be obtained by scanning these specimens with a fluorescence microscope. Since conventional diagnosis require amount of human efforts, detecting Tuberculosis bacilli automatically has drove many attentions [1, 2], 3]. All of these systems use a specific segmentation method to highlight the bacilli regions from the original image.

However, all these methods are based on statistical analysis of the profile and color information of bacilli, so they have disadvantages: Poor generalization ability. Stained M. tuberculosis bacilli display in images with varying appearances. Thus in 1], 2], 3], both color and contour features have been considered. But, statistical knowledge about the shape and color of the labeled bacilli is limited to dataset which is sensitive to experimental conditions such as luminance and camera types. Poor ability dealing with outliers. The contrast between bacilli and the background is regularly manifest given that stained bacilli fluoresce in the range between green and yellow up to white. In this case, color segmentation might be able to produce desired results. Nevertheless, when one bacteria

\footnotetext{
* This work has been partly supported by Ministerio de Educación of Spain (projects 'DEIPRO, id. TEC2009-14504-C02-01, and 'COMONSENS', id. CSD2008-00010).
} 


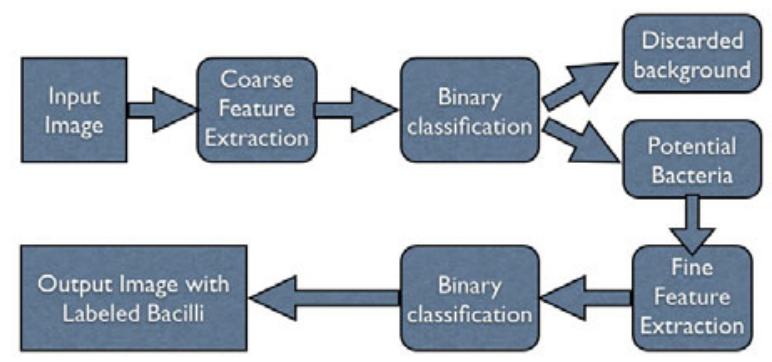

Fig. 1. The input original image passes through a front-end quick background subtraction system and drops most of background content. The filtered image (i.e., retained potential bacteria region) will be reanalyzed and reclassified with the purpose of improving the specificity of the whole system.

is overlapped by other debris objects such as cells, or when images have been taken under inapplicable luminance conditions, similar segmentation attempts may be proved futile.

Instead of getting involved in intricate segmentation algorithm investigation, we propose a quick background subtraction system as a front-end of the M. tuberculosis bacillus detection system shown in Fig 1 . This background elimination system is termed as a coarse feature descriptor followed by a binary classification (i.e., bacillus or background). The essential idea of designing such a front-end is to reduce the computational complexity while maintaining elevated sensibility and compensatory specificity. As the majority of the original image has been disposed by the front-end processor, steps of locating bacilli are only required to take on the retained possible regions which will dramatically accelerate the whole detection process. In section 2, four feature extraction algorithms: SIFT, color SIFT, Haralick Features and Histograms of Colors are modified to suit our M. bacillus recognition task. Section 3 gives experimental results based on real fluorescence images.

\section{Feature Extraction Algorithms}

Patches are extracted by sliding a local window across the image and classified as positive (i.e., presence of object) or negative (i.e, absence of object). Unlike articulated objects (e.g., pedestrians), bacilli do not contain a lot of structural or intra-class variations, specially scale variations, thus, it is reasonable to use single fixed-size sliding window. Characters based on small sliding window are more discriminative, but computational expensive. On the contrary, big sliding window avoids expensive computations by allowing more flexible moving steps, however, as more background pixels have been included, the results are less reliable. Given that bacillus generally is of size up to $20 \times 20$, in our experiment the size of patches has been chosen as $36 \times 36$.

Patch classifier is chosen as SVMs with linear kernel [4, 5. 


\subsection{SIFT and Color Based SIFT}

The original SIFT 6 6 descriptor initials with a keypoint detector followed by a local image descriptor. Different with the general image matching problem, in our case, neither multiscale invariability nor distinctive points localization should be necessarily required. Smooth the $i$-th color component $I(x, y, i)$ of one image with a variable-scale Gaussian mask $G(x, y, \sigma)$,

$$
\begin{aligned}
L(x, y, i, \sigma) & =G(x, y, \sigma) * I(x, y, i), \quad 1 \leq i \leq c . \\
G(x, y, \sigma) & =\frac{1}{2 \pi \sigma^{2}} e^{-\left(x^{2}+y^{2}\right) / 2 \sigma^{2}},
\end{aligned}
$$

where $*$ is a convolution operator.

Then the gradient of the Gaussian mask smoothed image can be written as

$$
\begin{aligned}
J(x, y, i) & =\nabla[L(x, y, i, \sigma)]=\nabla[G(x, y, \sigma) * I(x, y, i)] \\
& =[\nabla G(x, y, \sigma)] * I(x, y, i), \quad 1 \leq i \leq c .
\end{aligned}
$$

where $\nabla$ is the derivative operator, $\sigma$ indicates the width of the Gaussian and determines the quantity of smoothing (i.e., the gradient can be obtained by convolving the image with the horizontal and vertical derivatives of the Gaussian kernel function).

SIFT features for the $i$-th color channel of the patch $p(u, v ; c)$ are formed by computing the gradient $J(u, v, i)$ at each pixel of the patch. The gradient orientation is then weighted by its magnitude. This patch is then split into $4 \times 4$ grid, in each tile a gradient orientation histogram is formed by adding the weighted gradient value to one of the 8 orientation histogram bins.

An extension is CI-SIFT which embeds color information to conventional SIFT descriptor by applying the five color invariant sets presented in Table 3 of 7 .

\subsection{Histogram of Color}

Color signatures are extracted by using the histograms of color method proposed in 8 .

\subsection{Haralick Features}

Based on co-occurrence matrix, several statistics were proposed in 9], known as Haralick features.

Co-occurrence matrix of a $n$ bits gray-level image is of size $2^{n} \times 2^{n}$. One way to reduce the dimension of this matrix is to quantitate the image. The quantization for images of multidimensional color space can be done with $K$-means clustering algorithm. In the case of images of unit color component, uniform quantization can be employed. 


\section{Patches Classification Experiments}

\subsection{Datasets Description}

Images are required from a $20 \mathrm{x}$ fluorescence Nikon microscope with a prior automatic slide loader and a Retiga $2000 \mathrm{R}$ camera with acquisition time $0.6 \mathrm{sec}$, gain five and zero offset. For each patient, 200 RGB $1200 \times 1600$ (8 bits per color) images have been taken and stored. 4,987 bacilli of 952 images of 45 infected patients have been identified and labeled manually by experts. Patches centered at each bacteria haven been extracted. Furthermore, bacilli have been rotated 90, 180 and 270 grades so as to enrich the dataset. Finally, a positive dataset of 19,890 patches has been generated. Regarding the negative dataset, 100,031 negative patch samples haven been created by arbitrarily moving the local windows in images of a couple of healthy patients.

In the following experiments, 3,000 positive as well as negative randomly selected patches are used for training step and all the rest for test. Results are obtained by averaging 100 runs of a linear SVM. Due to space limitation, full detailed results can be found in technical report [10].

\subsection{Experiments}

Experiment E01: Without any feature descriptor. For the purpose of comparison, in this experiment none feature extraction processes have been taken. Patches are considered to be of size $20 \times 20$. PCA has been used to reduce the feature dimension. And, only the $B L U E$ component has been analyzed. As long as the patch is small enough, even without feature extraction, one simple linear-SVM classifier is capable to achieve a sensibility up to $93.6 \%$.

Experiment E02: SIFT. Both the sensibility and specificity are comparable regardless of the color spaces. For white color bacilli surrounded by yellow tissues, $B L U E$ is the only discriminative in the $R G B$ color space, which may explain why $B L U E$ component obtains the optimal results.

Experiment E03: CI-SIFT. In [7, the author stated that the degree of invariance of the five color invariant sets have the following relation: $E_{\omega}<W_{\omega}<$ $C_{\omega}<N_{\omega}<H_{\omega} . H_{\omega}$ which is the most robust to changes in imaging condition (see Table 1 in [7]) had the lowest discriminative power while $E_{\omega}$ which is the most sensitive to any changes achieved the best recognition precision.

Experiment E04: HF-without Quantization. It is always better not to normalize features sets obtained from the four adjacently measurements (see Fig. 2 of [10]) regardless of the implemented color spaces.

Experiment E05: HF-with Quantization. Quantization (see section 2.3) is firstly employed for each patch. 1,000 positive as well as negative patches have been picked randomly from the entire data set from which codebooks of colors are constructed with $K$-means clustering method. Regarding unit dimensional color spaces, uniform quantization method also has been studied. Excluding 
$R G B$, sensibility as well as specificity obtained in all the unit dimensional color spaces in this experiment are comparable to or even higher than those obtained in the previous experiment which reveals that quantizations are highly recommended. Furthermore, uniform quantization is much more reliable than $K$-means quantization.

\section{Conclusion and Future Line}

In this paper, we have analyzed the performance of several feature extraction algorithms. In Table 1 are the optimal cases for each algorithm. The $B L U E$ scale state-of-the-art SIFT-like descriptor outperforms the others. The specificity achieved by Histograms of colors method is more than $15 \%$ lower than the average level. CI-SIFT as well as HF take use both the geometrical and color signatures and are capable to obtain appealing results. Small patch may contain more discriminative signatures, but we aim to reduce the background as fast as possible which means that with the same accuracy bigger patches are always recommended. Since the computational complexity of most of attributes of the Haralick feature sets are $O$ (number of bits of the image ${ }^{2}$ ), the quantization step considerably accelerate the algorithm. Another conclusion we can make is that the $B L U E$ channel is the most robust compared with other color representations. By applying this background elimination process, approximated $96.92 \%$ background pixels can be subtracted from the original image. One example is given in Fig. 2,

One possible way to improve sensibility without degrading specificity is to use a different evaluation function (such as F-score, AUC) of the linear SVM classifier or add other simple implemented characters into the feature set.
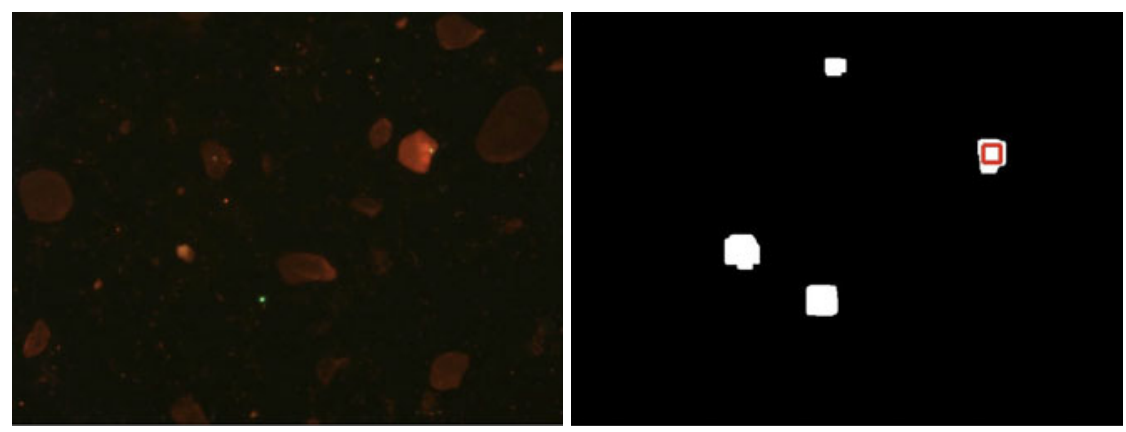

Fig. 2. The left figure is a fluorescence image with one bacteria. The right figure is the filtered image in which the white regions are detected potential bacilli locations, with the real bacteria bounded by the red rectangular for purpose of reference. Parameters used here are: SIFT descriptor on green color channel with step of the moving window equal to 1 . Most of the pixels of the image have been classified as background (i.e., the black regions). 
Table 1. Comparison of all the algorithms

\begin{tabular}{|c|c|c|c|c|c|c|}
\hline \multirow[t]{2}{*}{ method } & \multirow[t]{2}{*}{ parameters } & \multirow[t]{2}{*}{ sensibility } & \multirow[t]{2}{*}{ specificity } & \multicolumn{3}{|c|}{$\begin{array}{l}\text { computational complexity (approx- } \\
\text { imated number of executions per } \\
\text { patch) }\end{array}$} \\
\hline & & & & + & $x$ & others \\
\hline SIFT & Blue & $95.199(0.187)$ & $97.234(0.151)$ & $11 l^{2}$ & $12 l^{2}$ & $2 l^{2}$ \\
\hline \multirow[t]{2}{*}{ HoColor } & RGB, NumBin $=8$ & $93.418(0.357)$ & $79.672(0.464)$ & 0 & 0 & $3 l^{2}$ \\
\hline & BLUE, NumBin $=8$ & $91.097(0.484)$ & $75.931(0.525)$ & 0 & 0 & $l^{2}$ \\
\hline CI-SIFT & $E_{\omega}$ & $94.000(0.272)$ & $96.949(0.167)$ & $30 l^{2}$ & $31 l^{2}$ & $2 l^{2}$ \\
\hline \multirow[t]{2}{*}{$\mathrm{HF}$} & $\begin{array}{l}\text { RGB, no normaliza- } \\
\text { tion }\end{array}$ & $95.260(0.312)$ & $93.704(0.256)$ & $75 k^{2}$ & $66 k^{2}$ & $21 k^{2}$ \\
\hline & $\begin{array}{l}\text { BLUE, no normaliza- } \\
\text { tion }\end{array}$ & $93.792(0.232)$ & $93.409(0.218)$ & $25 k^{2}$ & $22 k^{2}$ & $7 k^{2}$ \\
\hline $\mathrm{HF}$ & $\begin{array}{l}\text { BLUE, codebook = } \\
8, \text { uniform Quantiza- } \\
\text { tion, no normaliza- } \\
\text { tion }\end{array}$ & $94.845(0.256)$ & $92.577(0.272)$ & $l^{2}+25 k_{Q}^{2}$ & $l^{2}+22 k_{Q}^{2}$ & $6 k_{Q}^{2}$ \\
\hline $\begin{array}{l}\text { None feature } \\
\text { extraction }\end{array}$ & $\begin{array}{l}\text { BLUE, patch size }= \\
20 \times 20 \text {, feature vector } \\
\text { is of size } 1 \times 400\end{array}$ & $93.617(0.307)$ & $97.046(0.189)$ & 0 & 0 & 0 \\
\hline
\end{tabular}

\section{References}

1. Osman, M.K., Mashor, M.Y., Saad, Z., Jaafar, H.: Colour image segmentation of Tuberculosis bacillus in Ziehl-Neelsen-Stained tissue Images using moving KMean clustering procedure. In: 4th Asia International Conference on Mathematical/Analytical Modelling and Computer Simulation, pp. 215-220. IEEE Press, Washington, DC (2010)

2. Foreo, M.G., Gristóbal, G., Alvarez-Borrego, J.: Automatic identification techniques of tuberculosis bacteria. In: Proceedings of the Applications of Digital Image Processing XXVI, San Diego, CA, vol. 5203, pp. 71-81 (2003)

3. Foreo, M.G., Sroukeb, F., Cristóbal, G.: Identification of tuberculosis bacteria based on shape and color. J. Real-Time Imaging 10(4), 251-262 (2004)

4. Cortes, C., Vapnik, V.: Support-vector network. Machine Learning 20, 273-297 (1995)

5. Chih-Chung, Lin, C.-J.: LIBSVM: A library for Support Vector Machines, http://www.csie.ntu.edu.tw/ cjlin/papers/libsvm.pdf

6. Lowe, D.G.: Distinctive image features from scale-invariant keypoints. Int. J. of Computer Vision 60, 91-110 (2004)

7. Geusebroek, J.M., Boomgaard, R.V.D., Smeulders, A.W.M., Geerts, H.: Color invariance. J. IEEE Transactions on Pattern Analysis and Machine Intelligence 23(12) (2001)

8. Swain, M.J., Ballard, D.H.: Color indexing. Int. J. of Computer Vision 7(1), 11-32 (1991)

9. Haralick, R.M., Shanmugan, K., Dinstein, I.: Textural features for image classification. IEEE Transactions on Systems, Man, and Cybernetics SMC-3, 610-662 (1973)

10. Gong, S., Artés-Rodrigués, A.: Science report, http://www.tsc.uc3m.es/ concha/TecReportBacilos.pdf 